\title{
Qualitätskriterien für Förderansätze zur Prävention von Rechenschwäche
}

\author{
Kristin Krajewski ${ }^{1}$, Stefanie Simanowski ${ }^{2}$ \\ Pädagogische Hochschule Ludwigsburg \\ 2 Universität Gießen
}

\begin{abstract}
Zusammenfassung: Die Förderung von Kindern mit schwachen mathematischen Basiskompetenzen ist eine wichtige Aufgabe, um diese Kinder vor der Entstehung einer Rechenschwäche zu bewahren. Selbst für Fachkräfte ist es aber nicht immer leicht, sich in der Vielfalt von Ansätzen und Materialien zur frühen Mathematikförderung zurechtzufinden. Ziel des Beitrags ist es daher, dem Praktiker einerseits inhaltliche Kriterien für Förderansätze in die Hand zu geben, die aus entwicklungs-, lern- und gedächtnispsychologischer Sicht die Wahrscheinlichkeit eines Fördererfolgs erhöhen. Andererseits werden auch Richtlinien gegeben, um das wichtige Kriterium des Wirksamkeitsnachweises von Förderansätzen zu beurteilen. Schließlich werden verfügbare Förderprogramme und gegebenenfalls vorliegende Evaluationsergebnisse hinsichtlich dieser Kriterien beurteilt.
\end{abstract}

Schlüsselwörter: Mathematische Förderung, Training, Evaluation, Effektivität, Kriterien

\begin{abstract}
Quality criteria for training approaches to prevent mathematical difficulties Summary: Fostering children with weak basic numerical competence is important in order to prevent these children from the development of mathematical difficulties. Even for professionals, however, it is not always straightforward to overlook the diversity of approaches and materials for early mathematical training. Hence, the aim of this article is to provide the practitioner with substantive criteria for training approaches. These criteria, based on the psychological perspective of development, learning and memory, are supposed to increase the likelihood of training success. Furthermore, guidelines to assess the important criterion of empirical evaluation of training effectiveness are given. Finally, available trainings are evaluated with regard to these criteria and the empirical evaluation at hand.
\end{abstract}

Keywords: Mathematical training, evaluation, effectiveness, criteria, mathematical difficulties

E ine zunehmend große Zahl an empirischen Studien im nationalen und internationalen Raum belegt die Bedeutung von mathematischen Basiskompetenzen für die schulischen Mathematikleistungen. Dabei endet der Einfluss der sogenannten Basiskompetenzen nicht etwa im Grundschulalter, sondern sie sind auch noch für die Mathematikleistungen in der Sekundarstufe von fundamentaler Bedeutung (Ennemoser et al. 2011). Aus dieser Erkenntnis heraus wurden Förderansätze für den Kindergarten sowie die Vor- und Grund- schule entwickelt, die der Entstehung von Rechenschwäche entgegenwirken sollen. Der größte Teil der vorhandenen Förderansätze zielt zu Recht auf die Förderung des Zahlverständnisses ab. Neben dieser Gemeinsamkeit basieren die Verfahren allerdings auf unterschiedlichen Definitionen und Theorien zum Konstrukt Zahlverständnis. Sie unterscheiden sich demzufolge auch in der Vorgehensweise des Förderns und berücksichtigen mehr oder weniger gut die begrenzten Fähigkeiten in Arbeitsgedächtnis, Aufmerksamkeit und Selbst- 
regulation von sogenannten "Risiko"-Kindern, welche besonders anfällig sind, eine Rechenschwäche zu entwickeln. Die Verfahren sind zudem nicht gleichermaßen effektiv oder es liegen in weiten Teilen keine tragfähigen empirischen Belege für deren Wirksamkeit vor.

Der vorliegende Beitrag soll Praktikern als Orientierungshilfe bei der Auswahl von mathematischen Förderansätzen dienen. Dabei wird zunächst auf inhaltliche Kriterien für die Qualität einer mathematischen Förderung und anschließend auf Kriterien zur empirischen Evaluation von Förderansätzen eingegangen.

\section{Inhaltliche Anforderungen an Förderansätze}

\subsection{Inhaltsspezifität}

Mathematische Förderung sollte inhaltsspezifisch sein, d.h. sie sollte sich unmittelbar auf mathematische Inhalte und nicht auf unspezifische Fähigkeiten wie etwa auf Wahrnehmung oder motorische Fähigkeiten konzentrieren (Moser-Opitz/Schmassmann 2003). Denn zum Aufbau eines tiefen Zahlverständnisses braucht es die Auseinandersetzung mit numerischen Inhalten, also mit Mengen und Zahlen. Auch das Klassifizieren, Diskriminieren, Sortieren und Ordnen von Dingen oder Erstellen und Finden von Mustern stellt keine mathematikspezifische Förderung dar, wenn hierbei nicht gleichzeitig ein direkter Bezug zu Mengen und Zahlen hergestellt wird.

\subsection{Entwicklungsorientierung}

\section{Orientierung an der natürlichen Entwicklungsabfolge}

Eine Förderung sollte sich an Theorien zur natürlichen Entwicklung mathematischer Basiskompetenzen orientieren und beim systemati- schen Aufbau dieser Kompetenzen keine Entwicklungsschritte auslassen. Das bedeutet, dass beispielsweise zunächst die Zahlwortkenntnis gefestigt und ein Verständnis für die Verknüpfung von Zahlen und Größen geschaffen werden muss, bevor Zahlen beim Rechnen überhaupt miteinander in Relation gesetzt werden können. Denn ein fehlendes Verständnis für die Verknüpfung von Zahlwörtern mit Mengen und Größenbegriffen kann als Kerndefizit von Kindern mit Rechenschwäche betrachtet werden (Krajewski/Ennemoser 2013). Die ZahlGrößen-Verknüpfung sollte daher besonders im Fokus der Förderung stehen, bevor der Aufbau von Rechenkompetenzen in Angriff genommen werden kann.

\section{Am nächsthöheren Entwicklungsniveau orientierte Darstellungsmittel}

Gleichsam sollte die Förderung die nächsthöheren Entwicklungsstufen immer mit im Blick haben. Dies gilt insbesondere für die verwendeten Fördermaterialien, die so ausgewählt sein sollten, dass sie nicht nur dem Erwerb niedrigerer Kompetenzen (wie etwa Zählfertigkeiten oder der Zahl-Mengen-Verknüpfung), sondern auch jenem höherer (Basis-)Kompetenzen dienen. So können etwa verschiedenartige Elemente zur Repräsentation von Zahlen (z. B. zwei Sterne für die 2, drei Sonnen für die 3) allenfalls zur Veranschaulichung dienen, dass Zahlen Mengen repräsentieren (einfaches Zahlverständnis). Sie eignen sich jedoch keinesfalls, um auch die wichtige Erkenntnis für ein tiefes Zahlverständnis zu fördern, nämlich dass von einer Zahl zur nächsten Zahl immer eins hinzukommt (denn zwei Sterne und noch einer dazu ergibt keine drei Sonnen). Entwicklungsorientiert wäre es daher, von Anfang an identische Elemente zur Darstellung der jeweiligen Zahlen zu verwenden. Denn so können die Kinder zur Erkenntnis geführt werden, dass aufeinanderfolgende Zahlen auseinander hervorgehen. 


\section{Minimalistische Kompetenzzuschreibungen}

Wenn eine Förderung grundlegend wirksam sein soll, ist es von außerordentlicher Bedeutung, die kindlichen Kompetenzen nicht zu überschätzen. Folge einer Überschätzung wäre eine Förderung, die zu hoch ansetzt, basale Kompetenzen weiterhin unzureichend belässt und damit höheren Kompetenzen jegliches Fundament verwehrt. Kann ein Kind beispielsweise die Zahlwortreihe korrekt und schnell von zwanzig rückwärts aufsagen (und dabei womöglich auch korrekt immer ein Element antippen), bedeutet dies nicht zwangsläufig, dass es auch ein Verständnis für die Quantität von zwanzig Elementen hat, das Wort „zwanzig” also mit einer Anzahl verknüpft. Da es für das korrekte Aufsagen der rückwärtigen Zahlwortreihe ausreicht, diese quasi durch „Nachplappern" auswendig gelernt wiederzugeben, ist es nicht zulässig, daraus beispielsweise Schlüsse über ein vorliegendes Kardinal- oder gar Subtraktionsverständnis zu ziehen. Ausgehend von einer solchen Sichtweise sollten Kinder immer nur auf genau der Entwicklungsebene verortet werden, die zum Erreichen der gezeigten Leistung minimal notwendig und gleichzeitig hinreichend ist (Krajewski/Ennemoser 2013).

\subsection{Verwendung von Materialien und Vorgehensweisen, die Arbeits- gedächtnis, Aufmerksamkeits- steuerung und Selbstregulation entlasten}

Es wurde vielfach gezeigt, dass Kinder mit (drohenden) Lernschwierigkeiten teils gravierende Beeinträchtigungen in Arbeitsgedächtnis, Aufmerksamkeit und Selbstregulation aufweisen (z. B. Schuchardt et al. 2013, Toll et al. 2011). Um den begrenzten kindlichen Arbeitsgedächtnisund Aufmerksamkeitsressourcen gerecht zu werden, sollte Wissen so vermittelt und Übungen so gestaltet werden, dass das Arbeitsgedächtnis entlastet und Fähigkeiten zur Aufmerksamkeit und zur Selbstregulation nicht zu sehr beansprucht werden.
Dies geschieht erstens, wenn Instruktion und Darstellungsmittel eindeutig die wesentlichen numerischen Aspekte erkennbar machen (Krajewski/Ennemoser 2010). Gute Darstellungsmittel sind selbstredend und vermitteln äußerlich klar sichtbar und auf konkrete Weise den Aufbau des Zahlenraums, die Zuordnung von Zahlwörtern zu Mengen und die Größenverhältnisse der Zahlen (z. B. größere Zahlen werden nicht nur durch entsprechend mehr Elemente dargestellt, sondern sie vereinnahmen auch entsprechend mehr "Ausdehnung" bzw. „Raum“; also etwa die Vier doppelt so viel wie die Zwei; von einer zur nächsten Zahl kommt immer eins hinzu; „Kraft der Fünf“). Dies lässt Arbeitsgedächtnisressourcen frei werden, weil keine irrelevanten Informationen (z. B. Unterschiede in Form, Farbe, Beschaffenheit) oder gar konfligierende Informationen (z. B. eine kleine Anzahl großer Elemente vs. eine große Anzahl kleiner Elemente) mit verarbeitet, also als solche erkannt und "gehemmt" werden müssen. Wird beispielsweise zum Kennenlernen der Zahlen die kleine Zahl 2 etwa durch zwei große Teller, die große Zahl 10 aber durch zehn kleine Gummibärchen dargestellt, müssen Form, Farbe, Beschaffenheit und die unpassenden Größenverhältnisse von Gummibärchen und Tellern als numerisch irrelevante Merkmale erkannt und weggehemmt werden, um die dahinter stehende Anzahl erfassen zu können. Dies stellt eine Abstraktion der Situation dar, die für Kinder mit bisher fehlendem Zahlverständnis völlig unmöglich ist. Bei geeigneten Darstellungsmitteln hingegen ist eine solche Abstraktion gar nicht erforderlich, denn die Größenverhältnisse der Zahlen sind direkt sichtbar. Folglich können Kinder mit einer ungünstigen Ausgangslage (nur) mithilfe solcher Darstellungsmittel ein Zahlverständnis erwerben (z. B. die Zahl 10 ist größer als die Zahl 2, weil sie „mehr Fläche/Raum” einnimmt). Zudem besteht hier auch keine Notwendigkeit, sich wichtige, aber äußerlich nicht anschaulich repräsentierte Zahlen merken zu müssen, um mit diesen operieren zu können, da sie ja kon- 
kret dargestellt, also „sichtbar" sind. Aus gedächtnispsychologischer Sicht sollte es daher selbstverständlich sein, Kindern zu erlauben, die Darstellungsmittel (beispielsweise auch ihre Finger) zur Repräsentation von Anzahlen solange offen verwenden zu lassen, wie sie dies benötigen. Ziel einer Förderung sollte (zunächst) also nicht sein, ihnen die Darstellungsmittel und damit ihre Gedächtnisstützen - möglichst frühzeitig (bzw. zu früh) zu entziehen.

Zur Schonung von Gedächtniskapazität und Aufmerksamkeitsregulierung trägt zweitens auch ein weitgehender Verzicht auf eine zu alltagsnahe oder fantasiereiche kontextuale Einbettung der Förderinhalte bei (Hasemann/Stern 2002). Aus Ressourcenperspektive ist es also nicht von Vorteil, fantasiereiche Geschichten einzubauen, die nicht wesentlich zum numerischen Verständnis beitragen und die Gedanken der Kinder eher von den Zahlen wegführen. Vielmehr ist eine Förderung eher effektiv, wenn die Aufmerksamkeit ausschließlich auf numerische Inhalte gelenkt und Nebensächliches weggelassen wird (Krajewski/Ennemoser 2010). Andernfalls können die oben genannten erforderlichen Abstraktions- und Hemmungsprozesse den Aufbau eines adäquaten Zahlverständnisses behindern. Ganz im Gegensatzzu Bedenken, eine geringe kontextuale Einbettung könnte demotivierend wirken, ist aus dieser Sicht anzunehmen, dass eine kontextarme Aufgabe durch die beschriebenen positiven Effekte auf das Kompetenzerleben letztlich sogar positive Effekte auf die Motivation hat (Deci/Ryan 1985).

Schließlich wird drittens auch durch häufige Wiederholungen des Gelernten, die zur notwendigen Automatisierung basaler Fertigkeiten und Kompetenzen führen, Speicherkapazität frei (Krajewski/Ennemoser 2010). Hoch automatisiert sein sollten im Laufe der Entwicklung/Förderung etwa das Aufsagen der Zahlwortfolge, der Größenvergleich von Zahlen oder der in- und auswendige Abruf von (konzeptuell verstandenen!) Eins-plus-Eins-Aufgaben. Die dadurch frei werdende Speicherkapa- zität kann nun für die Beschäftigung mit den numerischen Inhalten von Situationen genutzt werden, wodurch nach und nach ein immer flexiblerer Umgang mit dem Zahlenraum entstehen kann. Erst jetzt ist eine Einbettung in immer komplexer werdende Kontexte (bzw. Textaufgaben) möglich und auch sinnvoll.

\subsection{Verwendung "mathematischer Sprache" zur gezielten Aufmerksamkeitslenkung}

Um die Aufmerksamkeit der Kinder mit einer beeinträchtigten mathematischen Entwicklung ausschließlich auf numerische Lerninhalte zu lenken, sollten gezielt entsprechende mathematische Begriffe verwendet werden. Dabei ist zu beachten, dass die Alltagssprache von der korrekten "mathematischen Sprache" abweichen kann. Deshalb muss beispielsweise erklärt werden, dass der Begriff "größere" Zahl nichts mit der physischen Größe der Elemente zu tun hat, sondern sich lediglich auf die größere Anzahl bezieht. Die alleinige Zurverfügungstellung von Material - auch wenn dieses die numerischen Aspekte der Zahlen gut herausstellt - wird daher als nicht ausreichend betrachtet, um (potenziell) rechenschwache Kinder angemessen zu fördern. Vielmehr sollte ganz im Sinne von Wygotski (1934) Förderung ausdrücklich auch sprachlich anleiten und dadurch den Fokus der Aufmerksamkeit auf das Numerische der Situation lenken.

\section{Anforderungen an eine empirische Evaluation von Förderansätzen}

Ein wesentliches Gütemerkmal eines Förderverfahrens ist der empirische Nachweis für seine Wirksamkeit. Um die Wirksamkeit zu belegen, sollten empirische Studien durchgeführt werden, in denen das zu evaluierende Förderprogramm unter kontrollierten Bedingungen eingesetzt wird. 
Bei der Planung einer solchen Studie sollte darauf geachtet werden, dass immer mindestens eine Kontrollgruppe einbezogen wird (Köller 2013). Üblicherweise findet bei Kindern auch ohne zusätzliche Förderung eine natürliche Kompetenzzunahme statt, sodass allein der (signifikante) Kompetenzzuwachs einer Fördergruppe von einem ersten zu einem zweiten Untersuchungszeitpunkt niemals als Beleg für die Wirksamkeit eines Trainings gelten kann. Dieser Zuwachs könnte ebenso gut allein durch die natürliche Entwicklung der Kinder zustande kommen (Köller 2013). Eine solche natürliche Entwicklung wird durch die Kontrollgruppe abgebildet. Daher muss die Fördergruppe zeigen, dass sie im Laufe der Förderung stärker in ihren Kompetenzen zulegt als die Kontrollgruppe. Idealerweise sollte auch belegt werden, dass die Fördergruppe zudem auch einer Gruppe von Kindern überlegen ist, die eine alternative Förderung und damit vergleichbare Zuwendung erhält. So spricht es für die besondere Wirksamkeit einer Förderung, wenn die geförderten Kinder im Vergleich zu Kindern mit einer alternativen Förderung größere Leistungszuwächse erreichen. Das heißt, die Leistungssteigerung basiert dann nicht lediglich auf der Zuwendung durch die Förderkräfte, sondern auf den Förderinhalten (Hager 2008). Darüber hinaus sollte durch Vortest-, Nachtest- und Follow-up-Untersuchungen (vor der Förderung, unmittelbar nach sowie mind. einige Monate nach Abschluss der Förderung; Köller 2013) untersucht werden, ob sich nicht nur unmittelbar nach dem Training ein Fördereffekt zeigt (kurzfristiger Effekt), sondern auch noch lange Zeit nach Abschluss der Förderung (langfristiger Effekt; Hager/Hasselhorn 2000). Um tatsächliche Kompetenzzuwächse abbilden zu können, ist es hier wichtig, dass zu allen Untersuchungszeitpunkten möglichst die gleichen Testverfahren verwendet werden (Strathmann/Klauer 2010), am besten Parallelversionen desselben Tests. Schließlich sollten sich auch Fördereffekte auf solche mathematischen Aufgaben(typen) und Materialien, beispielsweise aus Schulleis- tungstests, nachweisen lassen, die nicht direkt im Training gefördert wurden (Transfereffekt; Hager/Hasselhorn 2000). Denn dies ist schließlich das Zielkriterium, wenn es um die Prävention von Rechenschwäche geht. Besonders beeindruckend ist der Wirksamkeitsnachweis also dann, wenn die Förderung nicht nur kurzfristig, sondern langfristig stärkere Leistungssteigerungen bewirkt, die sich zudem auch tatsächlich auf die mathematischen Schulleistungen auswirken (transferieren).

Bei der Durchführung einer Evaluationsstudie sollte darauf geachtet werden, dass sowohl die Testverfahren als auch die zu evaluierende (und ebenso die alternative) Fördermethode manualgetreu und mit der angegebenen Gruppengröße sowie der angegebenen Alters- und Zielgruppe umgesetzt werden (Moosbrugger/ Höfling 2006; Bedingungskonstanz; Amelang/ Schmidt-Atzert 2006). Zudem sollten die Förderkräfte nicht (wie bei Friedrich/Munz 2004) gleichzeitig auch diejenigen sein, die die Vorund Nachtests zur Überprüfung der Fördereffekte durchführen (damit werden Erwartungseffekte ausgeschlossen; Ludwig 2008). Bei der Dokumentation ist darauf zu achten, dass die Gründe für einen möglichen Ausschluss von Versuchspersonen transparent gemacht werden.

Bei der Auswertung der Studiendaten ist schließlich zu beachten, dass bei den Analysen keine Kinder ausgeschlossen werden, nur weil sie auf das Training nicht angesprochen haben (wie z. B. bei Ehlert/Fritz 2016a) oder an einem der Messzeitpunkte nicht teilgenommen haben (Köller 2013). Erforderliche Analysen, die mit allen Kindern aller Gruppen durchzuführen sind, sind die Überprüfung der oben genannten kurz- und langfristigen Effekte auf die spezifisch trainierten Kompetenzen sowie von Transfereffekten auf schulische Mathematikaufgaben mit geeigneten statistischen Verfahren. Bei der Überprüfung der Effekte genügt es nicht, wenn die geförderte Gruppe „augenscheinlich“ mehr zugelegt hat. Es muss auch überprüft werden, ob 
dieser stärkere Zuwachs auch statistisch signifikant ist, also nicht lediglich im Bereich der üblichen Messungenauigkeiten oder der statistisch zu erwartenden Zufallsschwankungen liegt. Hierfür müssen neben Gruppenmittelwerten und Standardabweichungen natürlich auch die relevanten statistischen Kennwerte angegeben werden. Zum Wirksamkeitsnachweis bei gleichen Ausgangswerten in den Gruppen wird zum Beispiel typischerweise eine sogenannte Varianzanalyse mit Messwiederholung durchgeführt (Köller 2013). Bei dieser gibt es jeweils einen Haupteffekt Messzeitpunkt, einen Haupteffekt Gruppe und eine Wechselwirkung zwischen beiden. Wenn der Haupteffekt Messzeitpunkt signifikant wird, bedeutet das, dass sich die Kinder - unabhängig von der Gruppenzugehörigkeit - im Laufe der Zeit verbesserten. Wenn der Haupteffekt Gruppe signifikant wird, bedeutet das, dass sich die Gruppen ungeachtet des Messzeitpunktes (und etwaiger Fördermaßnahmen) unterscheiden. Wenn die Wechselwirkung Messzeitpunkt x Gruppe signifikant wird, bedeutet das, dass eine der Gruppen über die Messzeitpunkte hinweg bzw. im Verlauf der Zeit einen stärkeren Zuwachs aufwies als die andere(n) Gruppe(n). Diese Wechselwirkung ist bei Varianzanalysen mit Messwiederholung also die wichtige, relevante statistische Größe zur Beurteilung der Wirksamkeit einer Förderung, da hier nur die Signifikanz dieser Wechselwirkung anzeigt, ob sich die Fördergruppe durch die Förderung besser entwickelte als die andere(n) Gruppe(n) (Rasch et al. 2006).

Inwieweit Kennwerte relevant sind und ob sie tatsächlich die von den Autoren beschriebenen Fördereffekte belegen, können statistische Laien nicht immer einschätzen. Als zuverlässig können üblicherweise Studien gelten, wenn sie in Zeitschriften mit Peer-Review (also Begutachtung durch Experten) erschienen sind. Werden Wirksamkeitsstudien hingegen beispielsweise in Büchern oder auf Internetseiten veröffentlicht, liegt keine Kontrolle vor, ob die Studie den methodischen Standards entsprechend durch- geführt, ausgewertet und interpretiert wurde und damit den wissenschaftlichen Gesichtspunkten Genüge trägt. Leider finden sich nicht selten Aussagen zur Evaluation eines Trainings, bei denen die Dokumentation der relevanten Statistiken vollkommen fehlt und daher verbale Aussagen zu vorgefundenen Effekten substanzlos sind. Auch die bloße Aussage "wissenschaftlich evaluiert/überprüft" sagt nichts darüber aus, ob die Überprüfung auch tatsächlich eine signifikante Wirkung aufgezeigt hat oder nicht.

Bei der Interpretation von Studienergebnissen sollte schließlich beachtet werden, dass Unterschiede in Leistungszuwächsen, die sich als "nicht signifikant" erweisen, statistisch nicht bedeutsam sind, d. h. auf Zufall (z. B. der Tagesform der Kinder, Hitze oder Lärm am Testtag etc.) beruhen können und deshalb nicht als Fördererfolg interpretiert werden dürfen - selbst wenn die Betrachtung der absoluten Leistungswerte oder die Abbildung in einem Diagramm dies nahelegen. Aussagen über eine dennoch "schwache" Wirksamkeit etwa sind dann nicht zulässig (Köller 2013).

\section{3. Überprüfung von Förderverfahren mit Blick auf die vorgestellten Kriterien}

Neben den nachfolgend dargestellten Förderverfahren gibt es Berichte von weiteren, unveröffentlichten Verfahren und deren Evaluation (z.B. Peucker/Weißhaupt 2005, Quaiser-Pohl 2008, Koch/Ellinger 2016). Diese wurden in die folgenden Betrachtungen nicht mit aufgenommen, da es nicht möglich war, die konkreten Förderunterlagen einzusehen.

\section{Komm mit ins Zahlenland \\ (Friedrich et al. 2011)}

Das ganzheitlich ausgerichtete Programm ist für 3-bis 6-jährige Kinder entworfen worden mit dem Ziel, diese effektiv in schulrelevanten 
Kompetenzen zu fördern. Hierfür wird der Zahlenraum als "Lebensraum“ der Zahlen interpretiert und jeder Zahl ein fester "Wohnort" zugeordnet. Durch Zahlenpuppen und Zahlenmärchen werden die Zahlen personalisiert und beseelt. Aufgrund des explizit ganzheitlich angelegten Konzepts (die Förderung zielt auch auf musikalische und motorische Fertigkeiten) ist das Programm weder rein inhaltsspezifisch noch stringent am mathematischen Entwicklungsverlauf orientiert. Obwohl sich durchaus gute Darstellungsmittel finden lassen (Holzwürfel in aufsteigenden Anzahlen für aufsteigende Zahlen), gerät die explizit numerische Zahlbetrachtung zugunsten sozial-emotionaler, aber numerisch irrelevanter Zahldarstellungen wie z. B. den Personeneigenschaften einer Ziffer vollkommen in den Hintergrund. Beispielsweise ist nicht erkennbar, dass von der Drei, die als Fee mit drei Wünschen repräsentiert wird, zur Vier (eine Puppe mit vier Zöpfen) eins dazukommt. Vielmehr verschwinden drei Wünsche und es kommen vier Zöpfe hinzu. Die Zahlen gehen also nicht auseinander hervor, numerische Beziehungen zwischen den Zahlen sind nicht zu erkennen. Die Materialien sind somit weder geeignet, die Entwicklung eines tiefen Zahlverständnisses anzubahnen, noch dieses zu fördern. Insbesondere wird auch das kindliche Arbeitsgedächtnis durch die Fantasiegeschichten mit zahlreichen irrelevanten Informationen zusätzlich belastet. Es werden hohe Anforderungen an die kindliche Selbstregulationsfähigkeit gestellt, um das Märchenhafte der Zahlen als numerisch irrelevante Information „wegzuhemmen" und den dahinter stehenden Sinn der Zahlen zu erfassen. Kindern mit noch fehlendem Zahlverständnis, einer schwachen Aufmerksamkeitsregulation und der eingeschränkten Fähigkeit zur Hemmung von irrelevanten Reizen wird es bei der vielgestaltigen Vorgehensweise kaum möglich sein, die numerischen Inhalte der Situationen zu erkennen und daraus die Struktur der Zahlen abzuleiten und zu verstehen. Die Wirksamkeitsstudien an drei- bis sechsjährigen (normal entwickelten) Kinder- gartenkindern weisen auf allgemeinere, aber nicht mathematikspezifische Verbesserungen hin (Friedrich/Munz 2006). Verbesserungen werden lediglich auf basalem numerischem Niveau wie z.B. Ziffernkenntnis, Kenntnis der Zahlwortfolge und Eins-zu-eins-Zuordnungen, aber nicht im Bereich höherer Kompetenzen wie der Anzahlzerlegung berichtet (Pauen/Herber 2009). Bei letztgenannter Studie bleibt allerdings der Vergleich mit einer Kontrollgruppe aus, sodass die hier dokumentierten Verbesserungen ebenso gut auf die allgemeine Entwicklung wie die Förderung zurückgeführt werden könnten. Friedrich und Munz (2006) sprechen im Diskussionsteil ihrer Studie gar von langfristigen Transfereffekten auf die schulischen Mathematikleistungen von trainierten Kindern. Allerdings finden sich hierfür im gesamten Manuskript zur Evaluationsstudie keinerlei Daten und auch keine statistischen Kennwerte. Die Aussage über langfristige Transfereffekte des Zahlenlandprogramms entbehrt somit jeglicher Grundlage. In einer weiteren Studie zeigte das Training „Komm mit ins Zahlenland“ im Vergleich zu einer untrainierten Kontrollgruppe keine Effekte auf die mathematischen Basiskompetenzen der Kinder. Im Vergleich zu einem anderen mathematischen Training ( $M Z Z$; Krajewski et al. 2007) war es diesem sogar signifikant unterlegen (Krajewski et al. 2008). Die Wirksamkeit des Zahlenlandtrainings auf die mathematische Entwicklung ist somit nicht eindeutig belegt.

\section{Mathematik im Vorschulalter (Rademacher et al. 2009)}

Dieses Programm besteht aus 17 Einheiten, welche bei 5- bis 6-jährigen Vorschülern acht verschiedene kognitive Fähigkeitsbereiche fördern sollen. Einige davon sind inhaltsspezifisch mathematisch (Zahlbegriff, Mengenauffassung, einfache Rechenoperationen), andere allgemeiner kognitiver Natur (visuelle Differenzierungsfähigkeit, räumliches Vorstellen, Umgang mit Symbolen, Erfassen abstrakt-logischer Zusam- 
menhänge, Ursache-Wirkungsprinzipien). Die Bereiche werden von den Autoren zwar allesamt als notwendige Voraussetzungen für das Verstehen mathematischer Zusammenhänge deklariert. Es finden sich jedoch keine Verweise auf wissenschaftliche Studien, die dies klar belegen würden, und zudem keine klaren Bezüge zu einer Entwicklungstheorie. Auch beinhaltet das Programm keine Darstellungsmittel, die klar die Struktur des Zahlenraums aufzeigen und damit numerische Denk- und Gedächtnisprozesse unterstützen würden. Aufgaben, welche explizit Zahlwörter, Ziffern und zugehörige Mengen verknüpfen, sind kaum zu finden. Vielmehr werden relativ willkürlich Aufgaben aneinandergereiht, die keinen Bezug zueinander und keinen Bezug zu Zahlen erkennen lassen. Darunter finden sich zahlreiche physikalische Experimente, die Klassifikation von Objekten, die Bestimmung von Begriffen zur räumlichen Orientierung oder die symbolische Bedeutung von Verkehrsschildern. Die wenigen numerischen Aufgaben enthalten teils irrelevante und Arbeitsgedächtnisressourcen raubende Anteile. So ist es bei einer Aufgabe beispielsweise erforderlich, sich gleichzeitig drei Zahlwörter zu merken und die passende Anzahl an Muggelsteinen zu legen. Zwei weitere Aufgabenstellungen sind gänzlich irritierend: Zum einen sollen Karten mit einer bestimmten Anzahl an Punkten Gläsern mit verschieden vielen Perlen zugeordnet werden. Allerdings stimmt die Anzahl der Kartenpunkte (z.B. drei bzw. 31) nicht mit der Anzahl der Perlen (z. B. ca. zehn bzw. ca. $100)$ überein. Zum anderen sollen auch Karten mit Ziffern Gläsern mit Perlen zugeordnet werden. Wiederum passen die Ziffern (z.B. 1 bzw. 5) nicht zur Anzahl der Perlen in den Gläsern (z.B. ca. zehn bzw. ca. 100). Weder wird das mit der Aufgabe verfolgte Lernziel deutlich, noch zeigt diese eine Orientierung an dem, was in der späteren mathematischen Entwicklung folgt. Zur Evaluation des Programms findet sich eine Evaluationsstudie, welche allerdings nur unvollständig informiert und keine relevanten Kennwerte liefert (Rademacher et al. 2005). So wird der Erfolg des Programms an den Mittelwertunterschieden in den Kompetenzen zum Nachtest festgemacht, ohne dass Vortestunterschiede kontrolliert werden würden. Aussagen zu kurzfristigen Effekten sind daher nicht zulässig. Langfristige und Transfereffekte auf mathematische Schulleistungen werden nicht berichtet.

\section{Mathematik- und Rechenkonzepte im Vor- und Grundschulalter - Training (MARKO-T; Gerlach et al. 2013) sowie Mina und der Maulwurf (Gerlach/Fritz 2011)}

Die beiden Trainingsprogramme wenden sich an Kinder im Alter von 5-8 bzw. 4-8 Jahren. Zielt MARKO-T als Individualtraining mit 57 Sitzungen nur auf mathematisch entwicklungsverzögerte Kinder und den Zahlenraum bis 20, so ist das zweite Programm als Kleingruppentraining mit 48 Sitzungen konzipiert und grundsätzlich für alle 4- bis 8-Jährigen zum Kompetenzaufbau im Zahlenraum bis 10 gedacht. Beide Verfahren haben zum Ziel, grundlegende arithmetische Konzepte zu vermitteln, welche von den Autoren in einem Entwicklungsmodell mit fünf Entwicklungsniveaus (Fritz et al. 2013) verortet werden. Damit erfüllen sie das erste Kriterium einer theoretischen Orientierung an einer als relevant erachteten natürlichen Entwicklungsabfolge. Der Trainingsaufbau umfasst entsprechend jeweils eine Abfolge von fünf Bausteinen zu den Konzepten Zählzahl, ordinaler Zahlenstrahl, Kardinalität und Zerlegbarkeit, Enthaltensein und Klasseninklusion sowie Relationalität. Eine Ausnahme stellt der vorgeschaltete Baustein in "Mina und der Maulwurf" dar, welcher eher unspezifische Übungen wie z. B. Merkmale erkennen und sortieren, Reihenbildung und Mengenvergleiche fokussiert. Mathematische Begriffe werden in beiden Programmen im Wesentlichen adäquat eingesetzt, um Zahlen und Zahlbeziehungen zu verdeutlichen. Was das Kriterium der Ressourcenorientierung anbelangt, ist dieses nicht immer erfüllt. In beiden Programmen wird 
etwa bereits von Anfang an mit verdeckten Anzahlen gearbeitet. Hier müssen sich die Kinder jeweils eine verdeckte Anzahl von Elementen merken, bevor sie diese mit einer offen liegenden Anzahl anderer Elemente vergleichen können. Kann ein Kind die erste Anzahl aber nicht im Arbeitsgedächtnis verfügbar halten, wird es diese Aufgaben nicht lösen können und dadurch im Erwerb der geförderten Kompetenzen eher behindert. Die Förderinhalte werden zudem in Geschichten wie etwa mit dem Mistkäfer Marko eingebettet, welche teils sehr komplexe Sachaufgaben darstellen und es erschweren, den Situationen das numerisch Wesentliche zu entnehmen. Darüber hinaus erscheinen nicht alle Darstellungsmittel ideal. Haufen und Kugeltürme, die gestapelt (MARKO-T) - und nicht etwa in einer Reihe nebeneinander gelegt und verglichen - werden sollen, könnten insbesondere bei Kindern mit Beeinträchtigungen in Aufmerksamkeit und Arbeitsgedächtnis den unmittelbaren Vergleich von Anzahlen erschweren. In "Mina und der Maulwurf" wiederum werden kleine (An-)Zahlen durch wenige große Blätter und - anders herum - große (An-)Zahlen durch viele kleine Blätter repräsentiert. Dies läuft der Anforderung entgegen, Zahlen für einen erfolgreichen $\mathrm{Er}$ werb des Zahlverständnisses zunächst so zu repräsentieren, dass die räumliche Ausdehnung der Darstellungselemente auch dem GröBenwert der Zahlen entspricht (d.h. doppelte Flächenausdehnung für doppelte Zahlenwerte). Nicht zuletzt scheint es aus gedächtnispsychologischer Sicht ungünstig, dass die pädagogische Fachkraft während der Förderung mit großer Häufigkeit absichtlich Fehler machen und darauf setzen soll, dass ein förderbedürftiges Kind diese entdeckt. Dies kann dazu führen, dass die korrekten Inhalte mit häufig präsentierten Fehlern konkurrieren, nicht häufig genug wiederholt und dadurch falsche Ergebnisse automatisiert werden. Nicht zuletzt kann eine solche Vorgehensweise auch das Vertrauen in die mathematische Kompetenz der Fachkraft erschüttern.
Zur Evaluation der beiden Programme wurden vier Wirksamkeitsstudien veröffentlicht (Ehlert/ Fritz 2013, Ehlert/Fritz 2016 a, Ehlert/Fritz 2016 b, Langhorst et al. 2013). In diesen Arbeiten wird zwar von signifikanten kurz- und langfristigen Trainingseffekten gesprochen (teils sogar mit Transfer auf die schulischen Mathematikleistungen). Diese Effekte wurden jedoch statistisch gar nicht auf Signifikanz überprüft. D.h. zur Analyse der Daten wurde zwar eine geeignete Analysemethode ausgewählt (Varianzanalyse mit Messwiederholung). Die relevanten statistischen Kennwerte, die eine Beurteilung der erhofften Fördererfolge erlauben würden, werden von den Autoren aber nicht angegeben. So werden statt einer signifikanten Wechselwirkung Messzeitpunkt $\times$ Gruppe (= relevanter Kennwert bei den durchgeführten Analysen, siehe oben) für alle Analysen lediglich signifikante Haupteffekte der Gruppe berichtet. Diese werden - wie auch in einer aktuellen Evaluationsstudie von Koch und Ellinger (2016) - fälschlicherweise jeweils so interpretiert, als würden sie die Effektivität des Trainings nachweisen. Auf Basis der vorgelegten Kennwerte der Studien sind Aussagen, dass die Kinder (kurz-, langfristig) von einer Förderung mit "Mina und der Maulwurf" oder MARKO-T profitierten, jedoch gar nicht möglich. Lediglich im Handbuch zum MARKO-T (Gerlach et al. 2013) ist für eine weitere Evaluationsstudie mit 53 entwicklungsverzögerten 4- bis 9-jährigen Kindern auch die Angabe der Wechselwirkung zu finden. Diese legt nahe, dass dort ein neun- bis zwanzigstündiges Training mit MARKO-T im Vergleich zu Kontrollkindern, welche entweder kein Training oder ein Sozialtraining erhalten hatten, kurzfristige Effekte auf die trainierten mathematischen Kompetenzen nach sich zog, auch wenn hier versäumt wurde, detailliert anzugeben, welche der drei Gruppen welcher Gruppe gegenüber signifikant unterlegen war. Über den angegebenen kurzfristigen spezifischen Effekt hinaus werden von den Autoren weder langfristige Effekte noch Transfereffekte auf die schulmathematischen Leistungen berichtet. 


\section{Mengen, zählen, Zahlen}

\section{(MZZ; Krajewski et al. 2007)}

Das MZZ-Förderprogramm wurde für Kinder im Vorschulalter konzipiert, hat sich mittlerweile aber auch als Maßnahme zur Prävention von Rechenschwäche für die erste Klasse bewährt. Es ist in Kleingruppen von idealerweise vier bis sechs Kindern einsetzbar. Die Durchführung erstreckt sich über einen Zeitraum von acht Wochen mit drei halbstündigen Fördersitzungen pro Woche. Ziel der Förderung ist der Aufbau eines sicheren tiefen Zahlverständnisses. Hierfür werden zunächst Zahlen- und Ziffernkenntnis gesichert und Größenvorstellungen von Zahlen aufgebaut (einfaches Zahlverständnis), bevor höhere Kompetenzen vermittelt werden, die auf das Verständnis für die Beziehungen zwischen Zahlen zielen (Inhaltsspezifität mit Orientierung am Entwicklungsmodell der ZahlGrößen-Verknüpfung von Krajewski 2007). Die zugehörigen Materialien wie z.B. Karten von Zahlenstrahlen, Punkten, Würfeln und Fingern betreffen den Zahlenraum bis 10 und gehen für aufeinanderfolgende Zahlen direkt auseinander hervor. Sie sind konkret-anschaulich und bildhaft, aber dennoch reduziert auf den numerischen Aspekt. Dieser wird insbesondere auch in der Zahlentreppe fokussiert, bei der die exakten Größenunterschiede zwischen den Zahlen und damit die Zahlrelationen auch dreidimensional direkt "sichtbar" sind (z. B. ist der Klotz mit der Zahl 3 halb so groß wie der mit der Zahl 6). Besonderer Wert wird darauf gelegt, dass die Kinder die numerischen Beziehungen sprachlich beschreiben und reflektieren. Die Wirksamkeit des MZZ-Programms wurde in zahlreichen Studien überprüft. In allen Studien wurde zusätzlich zur MZZ-Gruppe mindestens eine Kontrollgruppe einbezogen. Zudem wurden in allen Studien mindestens signifikante kurzfristige Effekte auf die mathematischen Basiskompetenzen bestätigt. Mehrere Studien untersuchten zudem langfristige und Transfereffekte und konnten auch diese nachweisen. Durchführung und Auswertung der Studien entsprachen jeweils den oben genannten me- thodischen Anforderungen. Bei Kindergartenkindern führte die MZZ-Förderung sowohl im Vergleich zu einer untrainierten Kontrollgruppe als auch zu einer Förderung mit einem allgemeinen Denktraining und einer alternativen mathematischen Förderung zu einer stärkeren Verbesserung des Zahlverständnisses, welche langfristig noch ein halbes Jahr nach Trainingsende nachweisbar blieb (Krajewski et al. 2008). Kurzfristige Fördereffekte zeigten sich bei Vorklasse-Kindern, also Kindern, die vom regulären Schuleintritt zurückgestellt wurden (Hasselhorn/Linke-Hasselhorn 2013). Mehrfach konnten kurz- und langfristige spezifische Fördereffekte sowie ein langfristiger Transfer auf das Basisrechnen und die schulischen Mathematikleistungen bei rechenschwachen Erstklässlern nachgewiesen werden (Ennemoser/Krajewski 2007, Ennemoser et al. 2015, Sinner 2011). Vergleichbare Effekte zeigten sich auch für eine unterrichtsintegrierte Förderung (z. B. Ennemoser 2010). Schüler mit einer Lernbehinderung und Schüler mit geistiger Behinderung konnten zumindest kurzfristig von einer Förderung durch MZZ profitieren und zeigten Leistungsverbesserungen vor allem im Verständnis der Zahl-Größen-Verknüpfung (Kuhl et al. 2012, Sinner/Kuhl 2010), dem Meilenstein in der numerischen Entwicklung. Wie bei anderen pädagogisch-psychologischen Förderprogrammen auch, wird der hohe Grad an Standardisierung gelegentlich als Nachteil des MZZ empfunden. Konkret wird befürchtet, dass das Programm nicht genügend Freiräume lasse, um individuell auf das einzelne Kind einzugehen. Allerdings sind individuelle Anpassungen durchaus möglich; für vorgenommene Abweichungen liegen jedoch keine zuverlässigen Wirksamkeitsnachweise vor. Während kleinere individuelle Anpassungen wie etwa zusätzliche Wiederholungseinheiten vergleichsweise unproblematisch sein sollten, bergen tiefere Eingriffe in den systematischen didaktischen Aufbau (z. B. umfangreichere Abwandlungen in Instruktion und Material) die Gefahr, die Wirksamkeit der Maßnahme zu mindern. 


\section{Empfehlungen für die Förderpraxis}

Zu Beginn dieses Artikels wurden Kriterien für Förderansätze vorgestellt, welche aus entwicklungs-, lern- und gedächtnispsychologischer Sicht das Potenzial besitzen, der Entstehung einer Rechenschwäche effektiv entgegenzuwirken. Herausgestellt wurden unter anderem die Bedeutung eines systematischen Kompetenzaufbaus entlang der natürlichen Entwicklungsabfolge und die Verwendung geeigneter Darstellungsmittel. Ein gutes Darstellungsmittel ist eine äußerlich sichtbare Vorlage für die entstehende geistige Vorstellung des Zahlenraums, z. B. einer Art innerem Zahlenstrahl oder der Repräsentation von Größenvorstellungen der Zahlen. Der unreflektierte Einsatz von alltagsnahen Materialien hingegen kann den Aufbau einer klaren Vorstellung vom Zahlenraum erschweren. Denn hierbei wird der numerische Aspekt von irrelevanten Informationen begleitet, wodurch die begrenzten Kapazitäten von Arbeitsgedächtnis und Aufmerksamkeit schnell ausgelastet sein können. Auch der zu schnelle Verzicht auf konkret greif- und sichtbare Darstellungsmittel kann die begrenzten Gedächtnis- und Aufmerksamkeitsressourcen überstrapazieren und dadurch den Erwerb des Zahlverständnisses behindern. Für den Zahlaufbau sollten idealerweise Darstellungsmittel gewählt werden, deren numerische und räumliche Größe nicht im Gegensatz zueinander stehen. Außerdem erleichtert die wiederholte Verwendung identischer Darstellungsmittel, Symbole und Materialien sowie die Verwendung numerischer Begriffe eine Aufmerksamkeitsfokussierung auf den numerischen Inhalt. Neben den inhaltlichen Kriterien zur Auswahl geeigneter Förderansätze sollte ein besonderes Augenmerk darauf gelegt werden, ob die Wirksamkeit der jeweiligen Förderung wissenschaftlich untersucht und tatsächlich auch belegt wurde.

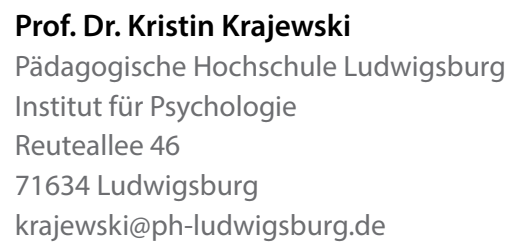

\section{Dr. Stefanie Simanowski}

Universität Gießen

Pädagogische Psychologie

Otto-Behaghel-Str. 10 F

35394 Gießen

\section{Literatur}

Amelang, M., Schmidt-Atzert, L. (2006): Psychologische Diagnostik und Intervention (4. Aufl.). Heidelberg: Springer, https://doi.org/10.1007/3-540-28507-5 Deci, E. L., Ryan, R. M. (1985): Intrinsic motivation and self-determination in human behavior. New York: Plenum, https://doi.org/10.1007/978-1-4899-2271-7

Ehlert, A.,Fritz, A. (2013): Evaluation of maths training programme for children with learning difficulties. South African Journal of Childhood Education 3 (1), 117-141, https://doi.org/10.4102/sajce.v3i1.34

Ehlert, A., Fritz, A. (2016 a): MARKO-T - Ein mathematisches Förderprogramm. In: M. Hasselhorn, W. Schneider (Hrsg.): Förderprogramme für Vor- und Grundschule, Tests und Trends - Jahrbuch der pädagogisch-psychologischen Diagnostik, Göttingen, Hogrefe, 29-48.

Ehlert, A., Fritz, A. (2016 b): Mina und der Maulwurf - Ein mathematisches Gruppentraining eingesetzt bei Kindern mit Sprachverständnisschwierigkeiten. In: M. Hasselhor, W. Schneider (Hrsg.): Förderprogramme für Vor- und Grundschule, Tests und Trends - Jahrbuch der pädagogisch-psychologischen Diagnostik, Göttingen, Hogrefe, 69-86.

Ennemoser, M. (2010): Training mathematischer Basiskompetenzen als unterrichtsintegrierte Maßnahme in Vorklassen. Empirische Pädagogik, 24, 336-352.

Ennemoser, M., Krajewski, K. (2007): Effekte der Förderung des Teil-Ganzes-Verständnisses bei Erstklässlern mit schwachen Mathematikleistungen. Vierteljahreszeitschrift für Heilpädagogik und ihre Nachbargebiete, 76, 228-240.

Ennemoser, M., Krajewski, K., Schmidt, S. (2011): Entwicklung und Bedeutung von Mengen-ZahlenKompetenzen und eines basalen Konventions- 
und Regelwissens in den Klassen 5-9. Zeitschrift für Entwicklungspsychologie und Pädagogische Psychologie, 43 (4), 228-242, https://doi.org/10.10 26/0049-8637/a000055

Ennemoser, M., Sinner, D., Krajewski, K. (2015): Kurz- und langfristige Effekte einer entwicklungsorientierten Mathematikförderung bei Erstklässlern mit drohender Rechenschwäche. Lernen und Lernstörungen, 4, 43 - 59, https://doi.org/10.1024/22350977/a000091

Friedrich, G., de Galgóczy, V., Schindelhauer, B. (2011): Komm mit ins Zahlenland, Freiburg, Herder.

Friedrich, G., Munz, H. (2004): Projekt- und Evaluationsbericht „Komm mit ins Zahlenland: Ein ganzheitliches Förderkonzept am Beispiel elementarer Mathematik". www.ifvl.de/material/ProjektberichtZahlenland.pdf, abgerufen am 27.1.2017.

Friedrich, G., Munz, H. (2006): Förderung schulischer Vorläuferfertigkeiten durch das didaktische Konzept „Komm mit ins Zahlenland“. Psychologie in Erziehung und Unterricht, 53, 134-146.

Fritz, A., Ehlert, A., Balzer, L. (2013): Development of mathematical concepts as basis for an elaborated mathematical understanding. South African Journal of Childhood Education, 3, 38-67.

Gerlach, M., Fritz, A. (2011): Mina und der Maulwurf. Frühförderbox Mathematik, Berlin, Cornelsen.

Gerlach, M., Fritz, A., Leutner, D. (2013): MARKO-T. Mathematik- und Rechenkonzepte im Vor- und Grundschulalter - Training, Göttingen, Hogrefe.

Hager, W. (2008): Evaluation von pädagogischpsychologischen Interventionsmaßnahmen. In: W. Schneider, M. Hasselhorn (Hrsg.): Handbuch der Pädagogischen Psychologie, Göttingen, Hogrefe, $721-732$

Hager, W., Hasselhorn, M. (2000): Einige Gütekriterien für Kriteriumsmaße bei der Evaluation von Interventionsprogrammen. In: Hager, W., Patry, J. L. (Hrsg.): Evaluation psychologischer Interventionsmaßnahmen. Standards und Kriterien: Ein Handbuch. Bern, Hans Huber, 169- 179

Hasemann, K., Stern, E. (2002): Die Förderung des mathematischen Verständnisses anhand von Textaufgaben - Ergebnisse einer Interventionsstudie in Klassen des 2. Schuljahres. Journal für MathematikDidaktik, 23 (3), 222 -242, https://doi.org/10.1007/ bf03338957

Hasselhorn, M., Linke-Hasselhorn, K. (2013): Fostering early numerical skills at school start in children at risk for mathematical achievement problems: A small sample size training study. Inter- national Education Studies, 6(3), 213-220,

https://doi.org/10.5539/ies.v6n3p213

Koch, K., Ellinger, S. (2016): Förderung sozial benachteiligter Kinder durch Förderung mathematischer Vorläuferkompetenzen - Evaluation des Programms „Kuno bleibt am Ball“ (KUBA). Zeitschrift für Heilpädagogik, 66, 513-525.

Köller, O. (2013): Evaluation pädagogisch-psychologischer Maßnahmen. In: E. Wild, J. Möller (Hrsg.): Pädagogische Psychologie, Heidelberg, Springer, 333-352.

Krajewski, K. (2007): Entwicklung und Förderung der vorschulischen Mengen-Zahlen-Kompetenz und ihre Bedeutung für die mathematischen Schulleistungen. In: G. Schulte-Körne (Hrsg.): Legasthenie und Dyskalkulie: Aktuelle Entwicklungen in Wissenschaft, Schule und Gesellschaft, Bochum, Winkler, 325-332.

Krajewski, K., Ennemoser, M. (2010): Die Berücksichtigung begrenzter Arbeitsgedächtnisressourcen in Unterricht und Lernförderung. In: H.-P. Trolldenier, W. Lenhard, P. Marx (Hrsg.): Brennpunkte der Gedächtnisforschung, Göttingen, Hogrefe, 337-365.

Krajewski, K., Ennemoser, M. (2013): Entwicklung und Diagnostik der Zahl-Größen-Verknüpfung zwischen 3 und 8 Jahren. In: M. Hasselhorn, A. Heinze, W. Schneider, U. Trautwein (Hrsg.): Diagnostik mathematischer Kompetenzen. Tests \& Trends N. F. 11, Göttingen, Hogrefe, $41-65$.

Krajewski, K., Nieding, G., Schneider, W. (2007): Mengen, zählen, Zahlen: Die Welt der Mathematik verstehen (MZZ). Berlin: Cornelsen.

Krajewski, K., Nieding, G., Schneider, W. (2008): Kurz- und langfristige Effekte mathematischer Frühförderung im Kindergarten durch das Programm „Mengen, zählen, Zahlen". Zeitschrift für Entwicklungspsychologie und Pädagogische Psychologie, 40, 135 - 146, https://doi.org/10.1026/0049-8637.40.3.135 Kuhl, J., Sinner, D., Ennemoser, M. (2012): Training quantity-number competencies in students with intellectual disabilities. Journal of Cognitive Education and Psychology, 11(2), $128-142$, https://doi. org/10.1891/1945-8959.11.2.128

Langhorst, P., Hildenbrand, C., Ehlert, A., Ricken, G., Fritz, A. (2013): Mathematische Bildung im Kindergarten - Evaluation des Förderprogramms „Mina und der Maulwurf" und Betrachtung von Fortbildungsvarianten. In: M. Hasselhorn, A. Heinze, W. Schneider, U. Trautwein (Hrsg.): Diagnostik mathematischer Kompetenzen. Tests und Trends, N. F. Bd. 11, Göttingen, Hogrefe, 113-134.

Ludwig, H. P. (2008): Erwartungseffekt. In: D. H. Rost (Hrsg.): Handwörterbuch Pädagogische Psychologie, Weinheim, Belz, 144-150. 
Moosbrugger, H., Höfling, V. (2006): Testdurchführung und -auswertung. In: F. Peterman, M. Eid (Hrsg.): Handbuch der Psychologischen Diagnostik, Göttingen, Hogrefe, 449-456.

Moser-Opitz, E., Schmassmann, M. (2003): Heilpädagogischer Kommentar zum Zahlenbuch 1. Hinweise zur Arbeit mit Kindern mit mathematischen Lernschwierigkeiten. Zug: Klett \& Balmer.

Pauen, S., Herber, V. (2009): Vom Kleinsein zum Einstein. Berlin: Cornelsen

Peucker, S., Weißhaupt, S. (2005): FEZ - Ein Programm zur Förderung mathematischen Vorwissens im Vorschulalter. Zeitschrift für Heilpädagogik (8), 300 - 305.

Quaiser-Pohl, C. (2008): Förderung mathematischer Vorläuferfähigkeiten im Kindergarten mit dem Programm „Spielend Mathe“. In: F. Hellmich, H. Köster (Hrsg.): Vorschulische Bildungsprozesse in Mathematik und Naturwissenschaften, Bad Heilbrunn, Klinkhardt, $103-125$.

Rademacher, J., Lehmann, W., Quaiser-Pohl, C., Günther, A., Trautewig, N. (2009): Mathematik im Vorschulalter. Göttingen: Vandenhoek \& Ruprecht.

Rademacher, J., Trautewig, N., Günther, A., Lehmann, W., Quaiser-Pohl, C. (2005): Wie können mathematische Fähigkeiten im Kindergarten gefördert werden? Ein Förderprogramm und seine Evaluation. Report Psychologie, 30, 366 - 374.

Rasch, B., Friese, M., Hofmann, W., Naumann, E. (2006): Quantitative Methoden Band 2: Einführung in die Statistik (2. Aufl.) (Kap. 7: Varianzanalyse mit Messwiederholung). Heidelberg: Springer.

Schuchardt, K., Grube, D., Mähler, C. (2013): „Schwierige Kinder" von Anfang an? Aufmerksamkeitsprobleme als Risikofaktor für die Schulfähigkeit. Kindheit und Entwicklung, 22(4), 217-223, https://doi.org/10.1026/0942-5403/a000120

Sinner, D. (2011): Prävention von Rechenschwäche durch ein Training mathematischer Basiskompetenzen in der ersten Klasse. Dissertation, Justus-LiebigUniversität Gießen.

Sinner, D., Kuhl, J. (2010): Förderung mathematischer Basiskompetenzen in der Grundstufe der Schule für Lernhilfe. Zeitschrift für Entwicklungspsychologie und Pädagogische Psychologie, 42 (4), 241 -251, https://doi.org/10.1026/0049-8637/a000 026

Strathmann, A. M., Klauer, K. J. (2010): Lernverlaufsdiagnostik: Ein Ansatz zur längerfristigen Lernfortschrittsmessung. Zeitschrift für Entwicklungspsychologie und Pädagogische Psychologie, 42 (2), 111 - 122, https://doi.org/10.1026/0049-8637/a000011

Toll, S., Van der Ven, H. G., Kroesbergen, E. H., Van Luit, J.E.H. (2011): Executive Functions as Predictors of Math Learning Disabilities. Journal of Learning Disabilities, 44 (6), 521 - 532, https://doi.org/10.1177/002 2219410387302

Wygotski, L. S. (1934, dt. Übers. 1974): Denken und Sprechen. Frankfurt: Fischer. 